This item was submitted to Loughborough's Research Repository by the author.

Items in Figshare are protected by copyright, with all rights reserved, unless otherwise indicated.

\title{
MDP-based MAC design with deterministic backoffs in virtualized 802.11
}

\section{WLANs}

PLEASE CITE THE PUBLISHED VERSION

http://dx.doi.org/10.1109/TVT.2015.2491932

PUBLISHER

(C) IEEE

VERSION

AM (Accepted Manuscript)

\section{PUBLISHER STATEMENT}

This work is made available according to the conditions of the Creative Commons Attribution-NonCommercialNoDerivatives 4.0 International (CC BY-NC-ND 4.0) licence. Full details of this licence are available at: https://creativecommons.org/licenses/by-nc-nd/4.0/

\section{LICENCE}

CC BY-NC-ND 4.0

\section{REPOSITORY RECORD}

Shoaei, Atoosa Dalili, Mahsa Derakhshani, Saeedeh Parsaeefard, and Tho Le-Ngoc. 2019. "Mdp-based MAC Design with Deterministic Backoffs in Virtualized 802.11 Wlans". figshare. https://hdl.handle.net/2134/21227. 


\title{
MDP-based MAC Design with Deterministic Backoffs in Virtualized 802.11 WLANs
}

\author{
Atoosa Dalili Shoaei*, Mahsa Derakhshani ${ }^{\dagger}$, Saeedeh Parsaeefard*, and Tho Le-Ngoc*
}

\begin{abstract}
This paper presents MAC protocols for a virtualized 802.11 network aiming to improve network performance and isolation among service providers (SPs). Taking into account the statistical properties of arrival traffic, a Markov Decision Process (MDP) is formulated to maximize the network throughput subject to SP reservations. By introducing the policy tree of the MDP, we present an optimal access policy. Each user can track this policy tree by carrier sensing and learn its transmission opportunity. As computational complexity of the policy tree grows exponentially with the total number of users, an efficient heuristic algorithm is proposed based on the MDP formulation where each user is assigned a deterministic backoff value. Numerical results show that performance of the proposed heuristic algorithm closely matches to the optimal policy. Moreover, both optimal and heuristic algorithms significantly improve TDMA and CSMA in terms of packet delivery ratio and isolation in unsaturated networks.
\end{abstract}

Keywords: Wireless local area networks (WLAN), virtualization, markov decision process (MDP), medium access control (MAC)

\section{INTRODUCTION}

In next-generation wireless networks, one promising solution to improve resource utilization and reduce implementation costs is wireless network virtualization (WNV), which enables sharing physical resources among different SPs [1]. WNV has been considered for different wireless local access networks (WLANs) [2]-[4]. In particular in 802.11-based WLANs, administrative virtualization is well established where a physical access point (AP) can advertise multiple service set identifiers, and thus can be shared by different SPs. Although such virtualization can differentiate flows from different SPs, it cannot further provide isolation among SPs.

In the context of WNV, isolation guarantees that any customization or reconfiguration in any specific SP could not disturb services provided to others. More specifically, any change in one SP (e.g., number of users and channel variations) should rarely impact resource utilization of others [5].

Manuscript received January 28, 2015; revised Jun 13, 2015; accepted September 19, 2015. The associate editor coordinating the review of this paper and approving it for publication was Y. Cheng.

Copyright (c) 2015 IEEE. Personal use of this material is permitted. However, permission to use this material for any other purposes must be obtained from the IEEE by sending a request to pubs-permissions@ieee.org.

A. Dalili Shoaei, S. Parsaeifard, and T. Le-Ngoc are with McGill University, Montreal, QC, Canada (email: atoosa.dalilishoaei@mail.mcgill.ca; saeideh.parsaeifard@mcgill.ca; tho.le-ngoc@mcgill.ca).

M. Derakhshani is with Imperial College, London, UK (email: m.derakhshani@imperial.ac.uk).
To provide isolation among SPs, one approach is exploiting deterministic resource allocation techniques such as time division multiple access (TDMA), in which an exclusive timeshare can be reserved for each SP. However, such allocation could lead to underutilization in unsaturated networks (users do not have always packets to transmit) since the reserved timeshare of a SP might partly be left unused in the presence of inactive users. On the other hand, random access protocols such as carrier sense multiple access (CSMA) are more opportunistic [6] and thus capable of adaptively managing the timeshare of each SP relative to its number of active users. Therefore, they can overcome utilization inefficiency. But, they suffer from isolation issues due to unavoidable collisions, which couple flows of different SPs [7]. These issues call for developing MAC protocols able to ensure QoS provisioning for each SP, which is the focus of this paper.

In conventional WLANs, for the purpose of QoS provisioning per user, IEEE 802.11e has proposed a polling-based MAC where AP transmits a polling frame (PF) to the intended user whenever it wants to receive/transmit a packet from/to that user [8]. Such polling-based MAC improves utilization efficiency and also avoids collisions compared to TDMA and CSMA, respectively. However, this mechanism has its own disadvantages and suffers from additional signaling overhead at each PF. Moreover, if the user cannot receive the PF, it would miss its transmission opportunity. To eliminate the need for PF transmission, [9] has proposed the deterministic backoff (DEB) method in which each user is assigned a different backoff value (BV) from others by AP. In DEB, all BVs are transmitted in a single beacon frame. This approach acts as the virtual polling via carrier sensing without the need for PF exchange. In DEB, a saturated traffic condition, i.e., each user always has a packet to transmit, is considered and thus deterministic BVs are assigned to different users in a round robin manner. Such an approach is not suitable for unsaturated traffic scenario and it reduces the resource utilization.

Different from conventional WLANs, the polling-based MAC in IEEE 802.11e and DEB cannot be directly applied in virtualized WLANs due to some technical challenges. First, instead of QoS per user, the predetermined QoS-requested by each SP-should be provided. Also, there is a need to keep the signaling overhead as low as possible. Finally, more realistic arrival traffic models need to be considered for users according to variety of emerging services where it is not practical to assume the saturated scenario for users. Addressing such challenges, in this paper, we aim to design a MAC 
protocol which takes into account dynamic nature of arrival traffic of users within SPs. We assume that user states evolve as a Markov model over superframes and AP is only aware of the transition probabilities. Under this assumption, a two-phase MDP-based MAC protocol is presented. In the first phase, a decision-making problem is formulated based on a MDP to develop an optimal polling mechanism. The design objective of this MDP is to maximize network throughput subject to SP reservations by applying a pricing mechanism.

In the second phase, we present an access scheme to virtually realize the proposed decision-theoretic polling mechanism. To this end, first, the optimal access policy is studied by introducing the policy tree. Then, a heuristic algorithm is proposed for deterministic backoff generation based on the MDP formulation. The aim is to avoid the need for transmitting the policy tree or frequent PFs. Through numerical results, we verify the performance of the developed MAC protocols in terms of packet delivery ratio, isolation, and throughput. In this paper, we quantify isolation capability of a MAC protocol in a WNV in terms of the fairness among SPs. More specifically, we measure such fairness in terms of equitable packet delivery ratios for different SPs relative to their reservations. It is confirmed that the proposed approaches noticeably improves the network throughput and provides better fairness among SPs in comparison with TDMA, CSMA and DEB.

The rest of this paper is organized as follows. We first introduce our system model in Section II. Then, Section III presents the MDP formulation and the optimal access policy. Subsequently, a heuristic access scheme is introduced in Section IV. Furthermore, Section V presents the numerical results. Finally, we conclude the paper in Section VI.

\section{System MODEL}

We consider an IEEE 802.11 WLAN with an AP which carries traffic belonging to $K$ different SPs. $\mathcal{N}_{k}$ denotes the set of users subscribed to SP $k$, where $\left|\mathcal{N}_{k}\right|=N_{k}$ is the number of users of SP $k$. The total number of all users in all SPs is $N_{\mathrm{u}}$, where $N_{\mathrm{u}}=\sum_{k=1}^{K} N_{k}$. Time is divided into fixed-length superframes (SF), each has one beacon frame followed by $T_{\mathrm{f}}$ time-slots as shown in Figure 1. In this setting, the smallest unit of time is called a backoff unit (BU). Each time-slot is equal to $T_{\mathrm{S}}$ BUs. $W=T_{\mathrm{f}} \times T_{\mathrm{s}}$ indicates the total number of BUs in a SF. The beacon frame is broadcasted by AP to users. It is assumed that each SP $k$ can reserve $r_{k}$ time-slots per SF.

The traffic generated at each user is described by an $\mathrm{ON}$ OFF Markov chain, which is a common model for bursty traffic sources [10], [11]. Each user $n_{k}$ of SP $k$ can be in two states at each SF $t, \theta_{n_{k}}(t)=0$ if it has no packet to transmit, and, $\theta_{n_{k}}(t)=1$ otherwise. User $n_{k}$ transits from state 0 to state 1 with probability $\alpha_{n_{k}}$ and stays in state 1 with probability $\beta_{n_{k}}$. During an ON period, it generates constant bit rate (CBR) data, i.e., $N_{p}$ packets per $\mathrm{SF}$, each with a length of $L_{p}$. If the user cannot access the channel before reception of the next beacon, the packet will be dropped. This model is applicable for a speaker in a voice call, where voice source alternates between talk $(\mathrm{ON})$ and silence (OFF) [12]. Moreover,

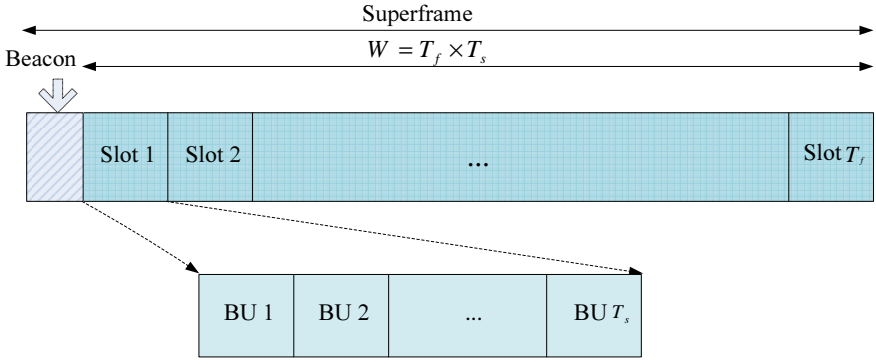

Fig. 1: Superframe structure of the proposed MAC for a virtualized 802.11 WLAN

this traffic model with $\beta_{n_{k}}=1$ can also represent periodic transmission. Realistic applications of this model can easily be found in the context of wireless sensor networks in which periodically taken samples from environment are transmitted to a data collector. Assuming that users transmit only new packets if variation has happened in the measurement, a bursty traffic model is more suitable than a periodic one [13].

We assume that wireless channel model includes path loss and small scale fading. The received SNR of user $n_{k}$ at AP is equal to $P_{t}\left|h_{n_{k}}\right|^{2} / \sigma^{2}$, where $P_{\mathrm{t}}$ is the transmission power, $\sigma^{2}$ is the noise power, and $h_{n_{k}}$ is the channel gain of the link from the user $n_{k}$ to the AP. More specifically, $h_{n_{k}}$ is equal to $C h_{n_{k}}^{\prime} d_{n_{k}}^{-\zeta / 2}$, where $d_{n_{k}}$ is the distance between user $n_{k}$ and the AP, $\zeta$ is the path loss exponent, $C$ is a constant dependent on the frequency and transmitter/receiver antenna gain, and $h_{n_{k}}^{\prime}$ represents the small scale fading component. For simplicity, without loss of generality, we normalize $C=1$ in the following discussions. If the received signal level falls below the receiver threshold, the receiver cannot decode the signal successfully. The probability that the received SNR is less than the receiver threshold $\psi$ (i.e., outage probability) is given by

$$
\phi_{n_{k}}=\operatorname{Pr}\left(\frac{P_{\mathrm{t}}\left|h_{n_{k}}^{\prime}\right|^{2} d_{n_{k}}^{-\zeta}}{\sigma^{2}} \leq \psi\right)=1-e^{-\frac{\sigma^{2} d_{n_{k}}^{-\zeta} \psi}{P_{\mathrm{t}}}} .
$$

Our main objective in this paper is to develop a MAC protocol for a virtualized 802.11 network which could maximize the network throughput, while considering isolation among SPs over each SF. To achieve these goals, we benefit both from carrier sensing as in CSMA and deterministic backoff generation as in DEB. In CSMA, since users pick $\mathrm{BVs}$ randomly, a collision may occur if two users pick the same BV. To avoid such collisions, in [9], a deterministic backoff generation scheme is proposed in which non-equal deterministic BVs are assigned to users by AP. For instance, if there are $N_{\mathrm{u}}$ users, AP assigns BVs from 1 to $N_{\mathrm{u}}$ to different users in a round robin manner. This information is broadcasted by AP to users in the beacon frame of each SF. The detailed description of CSMA and DEB protocols can be found in [14] and [9], respectively. In this work, we aim to use the idea of deterministic backoff generation to avoid collisions and ensure isolation among SPs. However, assuming that AP is not aware 
of user states at each SF and users are not saturated, assigning $\mathrm{BVs}$ in a round robin manner is not efficient. Thus, in process of BV generation, we take into account SP reservations and transition probabilities.

\section{MDP-BASED MAC DESIGN}

Here, we propose a virtual polling-based MAC protocol for a virtualized WLAN. First, we propose a decision-making problem to develop an optimal polling mechanism. Second, we present access schemes to virtually realize the proposed decision-theoretic polling mechanism with the aid of deterministic backoff generation, while avoiding frequent polling overheads.

A. MDP Formulation: To develop a polling mechanism, at each $\mathrm{BU}$ in a SF, AP must determine which user to poll. Let assume AP only knows the statistical properties of arrival traffic (i.e., $\alpha_{n_{k}} \& \beta_{n_{k}}$ ) and instantaneous user states (i.e., $\theta_{n_{k}}(t)$ ) at each SF are unknown. Since AP has partial knowledge, this decision-making problem can be formulated as a MDP with the following elements.

1) States: Let $s_{n_{k}}(t, b)$ be the transmission status of user $n_{k} \in \mathcal{N}_{k}$ in SF $t$ and BU $b$, where $b \in\{1, \ldots, W\}$. More specifically, $s_{n_{k}}(t, b)=1$ indicates that user $n_{k}$ is polled and transmitted successfully. $s_{n_{k}}(t, b)=-1$ represents that user $n_{k}$ is polled and has transmitted packet unsuccessfully due to outage. Furthermore, $s_{n_{k}}(t, b)=0$ means that the user is polled but it has no packet. Finally, $s_{n_{k}}(t, b)=-2$ means that user $n_{k}$ is not polled. Thus, the system state can be represented by $\boldsymbol{S}(t, b)=\left[\boldsymbol{S}_{k}(t, b)\right]_{k=1}^{K}$, where $\boldsymbol{S}_{k}(t, b)=\left[s_{n_{k}}(t, b)\right]_{n_{k}=1}^{N_{K}}$, for $n_{k} \in \mathcal{N}_{k}$.

2) Actions: An action is defined as which user is polled in the current BU. The index of user indicates the action and is denoted by $n_{a}(t, b)$. Note that $a(a=1, \ldots, K)$ represents the index of the SP for the selected user for polling at $t$, where the size of the action space is $N_{\mathrm{u}}$.

3) Transition Probabilities: The vector of transition probabilities between different states is represented by $\boldsymbol{P}=\left[P\left(\boldsymbol{S}, n_{a}, \boldsymbol{S}^{\prime}\right)\right]$, where $P\left(\boldsymbol{S}, n_{a}, \boldsymbol{S}^{\prime}\right)$ is the transition probability from state $S$ to $S^{\prime}$ taking action $n_{a}$. At each SF, $P\left(\boldsymbol{S}, n_{a}, \boldsymbol{S}^{\prime}\right)$ is zero except for

$$
\begin{aligned}
& P\left(\boldsymbol{S}, n_{a}, \boldsymbol{S}^{\prime}\right)= \\
& \begin{cases}\omega_{n_{a}}, & \text { if } s_{n_{a}}=-2,\left|s_{n_{a}}^{\prime}\right|=1, \text { and } s_{i}=s_{i}^{\prime}, \forall i \neq n_{a} \\
1-\omega_{n_{a}}, & \text { if } s_{n_{a}}=-2, s_{n_{a}}^{\prime}=0, \text { and } s_{i}=s_{i}^{\prime}, \forall i \neq n_{a},\end{cases}
\end{aligned}
$$

where $\omega_{n_{a}}$ represents the belief value of user $n_{a}$ at a given SF. In SF $t, \omega_{n_{k}}(t)$ is equal to the conditional probability (given the decision and observation history) that user $n_{k}$ has a packet to transmit and can be updated as

$$
\begin{aligned}
& \omega_{n_{k}}(t)= \\
& \left\{\begin{array}{l}
\beta_{n_{k}}, \quad \text { if } \quad\left|s_{n_{k}}(t-1, W)\right|=1 \\
\alpha_{n_{k}}, \quad \text { if } \quad s_{n_{k}}(t-1, W)=0 \\
\alpha_{n_{k}}\left(1-\omega_{n_{k}}(t-1)\right)+ \\
\beta_{n_{k}} \omega_{n_{k}}(t-1), \quad \text { if } s_{n_{k}}(t-1, W)=-2 .
\end{array}\right.
\end{aligned}
$$

If AP is aware that user $n_{k}$ had a packet in last SF (i.e., $\left.\left|s_{n_{k}}(t-1, W)\right|=1\right), \omega_{n_{k}}(t)$ is updated to $\beta_{n_{k}}$, which is the probability to stay in state 1 . If AP is aware that user $n_{k}$ had no packet to transmit (i.e., $s_{n_{k}}(t-1, W)=0$ ), $\omega_{n_{k}}(t)$ is updated to $\alpha_{n_{k}}$, which is the probability to transit from state 0 to 1 . Otherwise, if AP is not aware of user state (i.e., $\left.s_{n_{k}}(t-1, W)=-2\right), \omega_{n_{k}}(t)$ is updated to $\alpha_{n_{k}}\left(1-\omega_{n_{k}}(t-1)\right)+\beta_{n_{k}} \omega_{n_{k}}(t-1)$, which represents the conditional probability of having a packet in SF $t$ based on the law of total probability. A vector that consists of all belief values of different users is represented by $\boldsymbol{\Omega}(t)=\left[\omega_{n_{k}}(t)\right]$, which is called the belief vector.

4) Reward: The reward gained by the user $n_{a}$ polled in a BU is denoted by $R\left(\boldsymbol{S}, n_{a}, \boldsymbol{S}^{\prime}\right)$, while the system state was at $S$. Considering the SP reservations, $R\left(\boldsymbol{S}, n_{a}, \boldsymbol{S}^{\prime}\right)$ is defined as

$$
\begin{gathered}
R\left(\boldsymbol{S}, n_{a}, \boldsymbol{S}^{\prime}\right)=\left(1-\varphi_{n_{k}}\right) u\left(s_{n_{a}}^{\prime}\right)- \\
\gamma\left[\sum_{j \in \mathcal{N}_{a}} u\left(s_{j}^{\prime}\right)-r_{a}\right]^{+}+\gamma\left[\sum_{j \in \mathcal{N}_{a}} u\left(s_{j}\right)-r_{a}\right]^{+},
\end{gathered}
$$

where $r_{a}$ is the time-slot reservation of SP $a$, which user $n_{a}$ belongs to, $\gamma$ is a positive scalar, $u(x)=N_{p}$ if $|x|=1$ and $u(x)=0$ otherwise. Furthermore, $[x]^{+}=\max \{x, 0\}$. In (4), the first component counts the packet transmission if the polled user (i.e., user $n_{a}$ ) has a packet to transmit. The second and third components work as a pricing policy to avoid polling a user whose SP has already been assigned a sufficient number of time-slots according to its reservation.

5) Objective Function: The design objective is to maximize the reward over one SF, which consists of $W$ BUs. Thus, in SF $t$ and $\mathrm{BU} b$, a user is picked such that the expected total reward obtained over the remaining time of the SF is maximized. A policy $\pi_{t, b}(\boldsymbol{S})$ is a mapping from state $\boldsymbol{S}$ at BU $b$ in SF $t$ to an action. The optimal policy is the one that achieves the highest expected reward. To obtain the optimal policy, we refer to value function $V_{t, b}(\boldsymbol{S})$, which denotes the maximum expected remaining reward that can be accrued starting from $S$ and BU $b$ in SF $t$,

$$
\begin{aligned}
V_{t, b}(\boldsymbol{S})=\max _{n_{a}} & \sum_{\forall \boldsymbol{S}^{\prime} \neq \boldsymbol{S}} P\left(\boldsymbol{S}, n_{a}, \boldsymbol{S}^{\prime}\right) \times \\
& {\left[R\left(\boldsymbol{S}, n_{a}, \boldsymbol{S}^{\prime}\right)+V_{t, b+\Delta\left(\boldsymbol{S}, n_{a}, \boldsymbol{S}^{\prime}\right)}\left(\boldsymbol{S}^{\prime}\right)\right], }
\end{aligned}
$$

where $\Delta\left(\boldsymbol{S}, n_{a}, \boldsymbol{S}^{\prime}\right)=L_{p} \times N_{p}$ if $s_{n_{a}}^{\prime}=1$ and $\Delta\left(\boldsymbol{S}, n_{a}, \boldsymbol{S}^{\prime}\right)=$ 1 if $s_{n_{a}}^{\prime}=0$. Thus, the optimal policy can be obtained as

$$
\pi_{t, b}^{*}(\boldsymbol{S})=\arg \max _{n_{a}} V_{t, b}(\boldsymbol{S})
$$

To solve (6), one approach is the value iteration technique whose computational complexity is exponentially growing with $N_{\mathrm{u}}$ [15]. To overcome this issue, the optimal action can be derived on the basis of the highest expected immediate reward instead of expected remaining reward. In other words, at each $\mathrm{BU}$, the effect of action on future decisions can be neglected. In the following, we consider this reduced-complexity approach by optimizing only the expected immediate reward.

B. Optimal Access Policy: Based on the developed MDPbased polling mechanism, AP needs to inform the polled user 


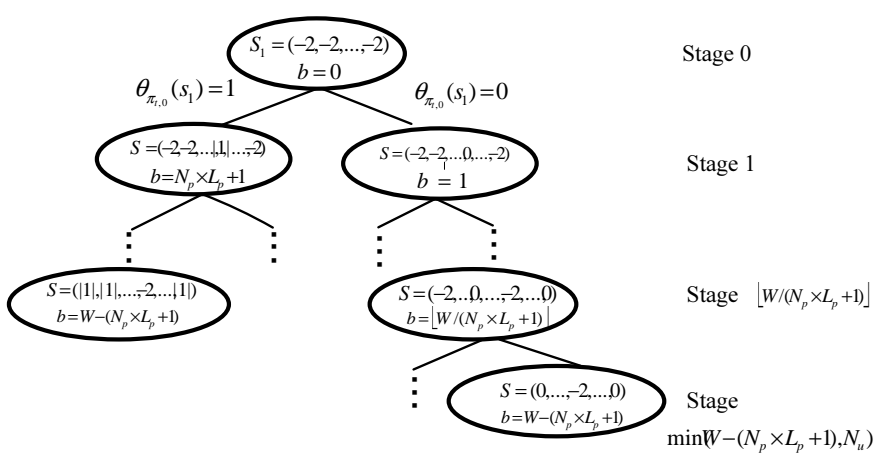

Fig. 2: Policy tree

by transmitting a PF, which causes signaling overheads. Here, we propose an access mechanism without PF transmission. In this approach, all required access information-optimal policies for all possible states-for each users can be transmitted in the beacon frame. We aim to transform this information in a way that users can access the channel only through carrier sensing. To this end, we exploit the policy tree, where each node represents one state and its corresponding action, i.e., which user should be polled. In this tree, from each node, at most two branches are originated; the left branch represents $\theta_{n_{k}}(t)=1$, i.e., the polled user has packet to transmit; and, the right one represents $\theta_{n_{k}}(t)=0$.

To access the channel with the help of information provided by the policy tree, each user can find out its transmission opportunity during a SF. To this end, each user has to sense the channel from the first BU and keep track of the channel state for each BU. Consequently, at each stage, the user can follow the polling path. Then, if the path leads to a node which corresponds to that user, if the user has a packet to transmit it would sense the channel for one BU and if it is detected as idle, transmission would happen in the next BU. In this binary tree, assuming that root lies in stage 0 , the first right branch terminates at stage of $\min \left(W-\left(N_{p} \times L_{p}+1\right), N_{\mathrm{u}}\right)$. Thus, the complexity of this algorithm is $\mathcal{O}\left(2^{\min \left(N_{\mathrm{u}}, W\right)}\right)$.

\section{Heuristic Algorithm for Backoff Generation}

For large values of $N_{\mathrm{u}}$, the size of policy tree becomes large, which results in a high computational complexity and large beacon frames. Therefore, in this section, we present a simple but efficient algorithm to implement virtual polling through deterministic backoff generation. In the optimal policy, at each decision time, the action with the highest expected immediate reward is selected. According to (4), the reward is equal to the throughput if the number of time-slots allocated to each and every SP is smaller than its reservation. Thus, under this condition, the optimal solution would be to choose users with the highest $Q_{n_{k}}=\left(1-\varphi_{n_{k}}\right) \times \omega_{n_{k}}$. Based on this intuition, to reduce complexity, we propose a heuristic algorithm as following.

At first, users are sorted according to their $Q_{n_{k}}$ descendingly. Then, at each step, we assign BVs to users which are started form 0 and incremented by 1 . The user with the highest

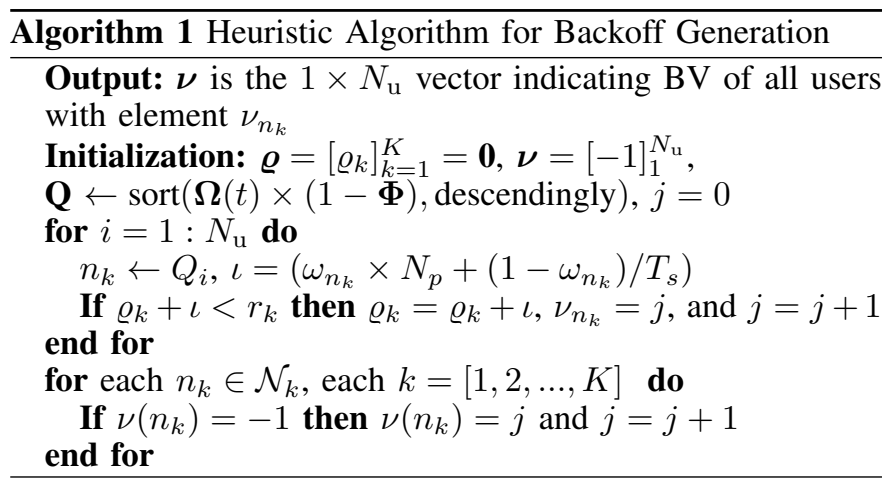

$Q_{n_{k}}$ will be selected at each step as long as the total time allocated to users belonging to the SP $k$ is smaller than $r_{k}$. But, since the moment that a selected SP is assigned a sufficient number of time-slots (equal to its reservation), the rest of users belonging to that SP will not be considered for backoff value allocation until all SPs would met their reservations. This is because the SP which its allocated time-slots exceeds its reservations will be penalized according to (4). Thus, next, the user from other SPs with the highest $Q_{n_{k}}$ among the remaining users will be chosen. When the number of allocated timeslots for all SPs exceeds their reservations, the BV assignment for the remaining users will continue according to their $Q_{n_{k}}$ descendingly.

In order to access the channel in this protocol, at each BU, if $\mathrm{BV}$ is equal to 0 , the user would sense the channel for one $\mathrm{BU}$ and transmit in the next BU. In case of busy channel, the packet would be dropped. Otherwise, it would decrease its BV by 1 . If the channel is sensed busy, the user would go to the sleep mode for $L_{p}$ BUs. Therefore, the user has to sense all BUs smaller than its BV except for those that it is in the sleep mode. It should be noted that the complexity of this algorithm is dependent on the sorting algorithm which is $\mathcal{O}\left(N_{\mathrm{u}} \log N_{\mathrm{u}}\right)$.

A drawback of this algorithm is that it requires users being able to synchronously count BUs. However, a user's backoff counter may possibly become out of synchronization due to the hardware clock imperfections inside the wireless network interface cards, which is commonly referred to as clock drift. If a user loses synchronization with others, it would drift to a new BU (other than the scheduled one). This BU can be possibly busy and collision can happen. Nowadays, this event is less concerned as it rarely occurs for commercial products [9].

\section{NUMERICAL RESULTS}

Here, we present numerical results to evaluate the performance of the proposed MDP-based MAC protocols in comparison with CSMA, DEB and TDMA-based MAC protocols in terms of packet delivery ratio, isolation, and throughput. For TDMA-based MAC, it should be noted that time-slots are first distributed among SPs based on their corresponding reservations. Within each SP $k$ at each SF, $r_{k}$ users with the highest $Q_{n_{k}}$ would be allocated to the time-slots. All algorithms are implemented in MATLAB and the results are 
based on the average of 100 repeated simulations over random distributions of users, each of which is 10,000 superframes long. It should be noted that based on simulation results the standard deviation of the sample mean is less than $1 \%$ when sample size is 10,000 SFs. We consider an uplink transmission in a virtualized WLAN serving 4 SPs in a circular area with a radius of $5 \mathrm{~m}$. Users are randomly distributed (from a uniform distribution) in this area. Each SP has a reservation of 4 timeslots (i.e., $r_{k}=4$ ). Furthermore, $T_{\mathrm{f}}=16, L_{\mathrm{p}}=T_{\mathrm{s}}=12$ BUs, $N_{\mathrm{p}}=1$, and $\gamma=0.8$. The channel parameters are set as path loss exponent $\zeta=3$, receiver threshold $\psi=0 \mathrm{~dB}$, and $\frac{P_{\mathrm{t}}}{\sigma^{2}}=20 \mathrm{~dB}$.

To study the impact of user distribution on the isolation and throughput achieved by the MAC algorithms, we consider an unsaturated network with two examples of balanced and unbalanced user distributions. For a balanced scenario, all SPs have the same number of users (i.e., $N_{k}$ ) and arrival traffic statistics (i.e., $\alpha$ and $\beta$ ). Let $N_{k}^{a}=N_{k} \times \lambda_{1}$ be the average number of active users for SP $k$, where $\lambda_{1}=\frac{\alpha}{1-\beta+\alpha}$ is the steady state probability of user being at state 1 . We assume that $N_{k}^{a}$ of each SP is equal to its reservation. In other words, we set $r_{k}=N_{k} \times \lambda_{1}$. This assumption represents a network, where its average load is equal its capacity. But, it should be considered that instantaneous number of active users at each SF could be larger or smaller than the reservation. Accordingly, we consider 4 different values of $(\alpha, \beta) \in\{(0.1,0.9),(0.2,0.6),(0.3,0.1),(0.1,0.6)\}$ for users. For each value of $(\alpha, \beta)$, we set $N_{k}=r_{k} / \lambda_{1}$, which results in $\{8,12,16,20\}$. For an unbalanced scenario, one SP has a larger number of users than other SPs. This scenario represents an overloaded network. For this scenario, we consider $(\alpha, \beta)=(0.1,0.9)$ for all users. Then, we set $N_{k}=8$ for $k \in\{1,2,3\}$ which ensures $r_{k}=N_{k} \times \lambda_{1}$, while $N_{4}$ can accept larger values. Thus, the average number of active users in SP 4 is larger than other SPs and its own reservation.

1) Packet Delivery Ratio: Packet delivery ratio (PDR) is defined as the ratio of number of packets that are successfully transmitted to total number of generated packets. Figure $3 \mathrm{a}$ shows the PDR versus the number of users per SP for the balanced scenario. As can be observed, the optimal and heuristic approaches achieve the same PDR. Comparing to CSMA, DEB, and TDMA, the proposed approaches improve the PDR performance. Nevertheless, it is shown that their performance is decreasing as $N_{k}$ increases, although the average number of active users is fixed to 4 . The reason is that the size of BVs assigned to the users are dependent on the number of users. With a larger $N_{k}$, larger BVs would be assigned to users which obviously causes more wasted time in waiting. On the other hand, the performance of CSMA is independent of $N_{k}$ considering that $N_{k}^{a}$ is fixed. This is because of the random backoff generation procedure in CSMA, which is affected by the number of active users, not the total number of users.

Unlike CSMA, which is only affected by $N_{k}^{a}$, the TDMA performance is more sensitive to the arrival traffic statistics. In particular, its PDR is generally decreasing with $\lambda_{1}$ since the probability that an assigned time-slot remains idle is

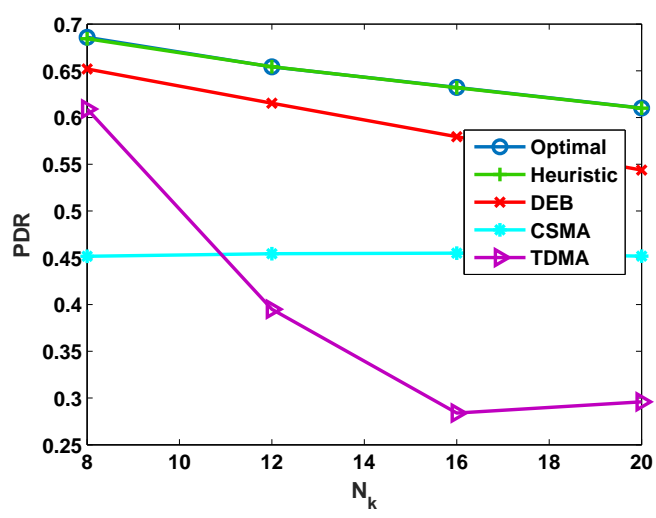

(a) PDR versus $N_{k}$, balanced scenario

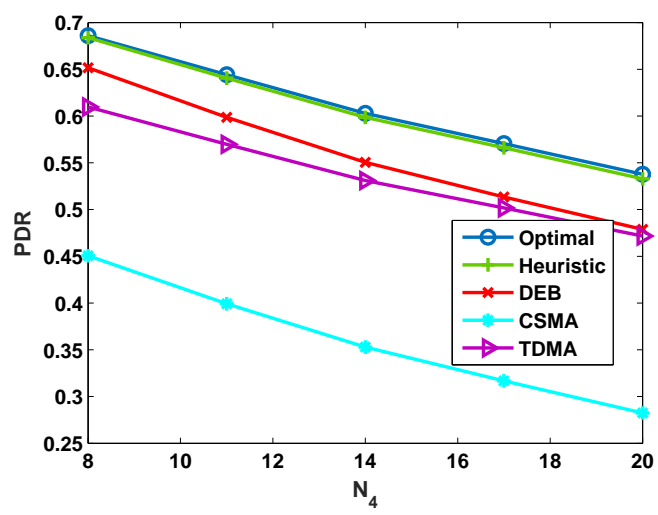

(b) PDR versus $N_{4}$, unbalanced scenario

Fig. 3: Total packet delivery ratio

decreased as shown in Figure 3a. However, it is shown that the TDMA performance increases for $N_{k}=20$ (corresponding to $(\alpha, \beta)=(0.1,0.6))$ compared to $N_{k}=16$ (corresponding to $(\alpha, \beta)=(0.3,0.1))$. This can be explained by the fact that TDMA performance depends on both $\lambda_{1}$ and $|\alpha-\beta|$. The reason is that the larger value of $|\alpha-\beta|$ provides higher probability to predict the user state given observation of user state. Therefore, the probability that an assigned time-slot remains idle would be decreased. Figure $3 \mathrm{~b}$ illustrates PDR versus $N_{4}$, for the unbalanced scenario. As expected, for all algorithms, PDR decreases by increasing $N_{4}$. This is because the number of generated packets of SP 4 is increasing with $N_{4}$. Moreover, compared to the balanced scenario, it is shown that the PDR improvement provided by the proposed MAC protocols relative to DEB is increased. This fact shows the effectiveness of using the belief vector in BV assignment instead of round robin assignment in DEB.

2) Isolation Index: We introduce an index to measure isolation among SPs based on Jain's fairness index [16]. To this end, first, we define $\rho_{k}$, PDR of SP $k$ considering its reservation, as

$$
\rho_{k}(t)=\min \left(\boldsymbol{A}_{k}(t) \boldsymbol{\theta}_{k}(t)^{T} / \min \left(\boldsymbol{\theta}_{k} \mathbf{1}^{T}, r_{k}\right), 1\right),
$$

where $\boldsymbol{\theta}_{k}(t)=\left[\theta_{n_{k}}(t)\right]_{n_{k}=1}^{N_{k}}$ represents the user states and 


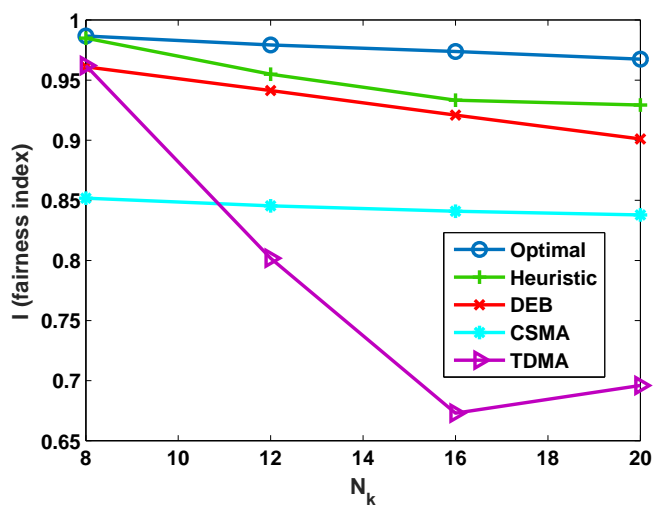

(a) Isolation versus $N_{k}$, balanced scenario

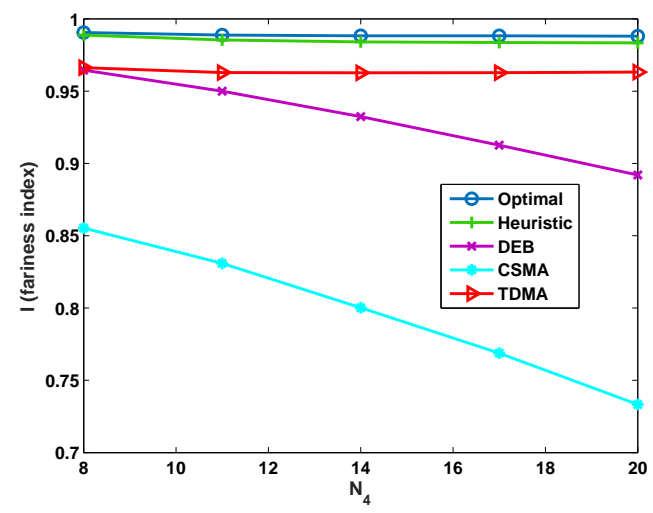

(b) Isolation versus $N_{4}$, unbalanced scenario

Fig. 4: Average isolation index

$\boldsymbol{A}_{k}(t)$ denotes the allocation strategy for SP $k$ in SF $t$. Thus, $\boldsymbol{A}_{k}(t) \boldsymbol{\theta}_{k}(t)^{T}$ represents the number of transmitted packets for SP $k$ in SF $t$. It should be noted that we limit the maximum number of generated packets of SP $k$ (i.e. $\boldsymbol{\theta}_{k} \mathbf{1}^{T}$ ) that potentially can be served, to its reservation $r_{k}$. This is the reason that $\min \left(\boldsymbol{\theta}_{k} \mathbf{1}^{T}, r_{k}\right)$ is used in the denominator in (7). In an ideal case, a MAC protocol would assign time to different SPs such that $\rho_{k}(t)=1$ regardless of number of active users. Thus, we measure the isolation level provided by a MAC protocol based on the Jain's fairness index, which can represent the variability among $\rho_{k}(t), \forall k$. We define the isolation index $I(t)$ in SF $t$ as $I(t)=\left(\sum_{k=1}^{K} \rho_{k}\right)^{2} /\left(K \sum_{k=1}^{K} \rho_{k}^{2}\right)$, where larger values of $I$ indicates better isolation among different SPs. Maximum value of $I$ is 1 which indicates that all SPs fairly have a time share of a SF relative to their reservations.

In Figure $4 \mathrm{a}, I$ is demonstrated versus the number of users per SP for the balanced scenario. Note that $I$ is measured for each SF and then the average over 10,000 SFs is plotted. It can be seen that the isolation index of the optimal policy is close to one. Except for CSMA, the isolation index decreases for all MAC schemes as $N_{k}$ increases. Similar to PDR, the isolation index of TDMA is dependent on $|\alpha-\beta|$. Thus, it is increasing form $N_{k}=16$ (corresponding to $(\alpha, \beta)=(0.3,0.1)$ ) to $N_{k}=20$ (corresponding to $(\alpha, \beta)=(0.1,0.6)$ ). Figure $4 \mathrm{~b}$

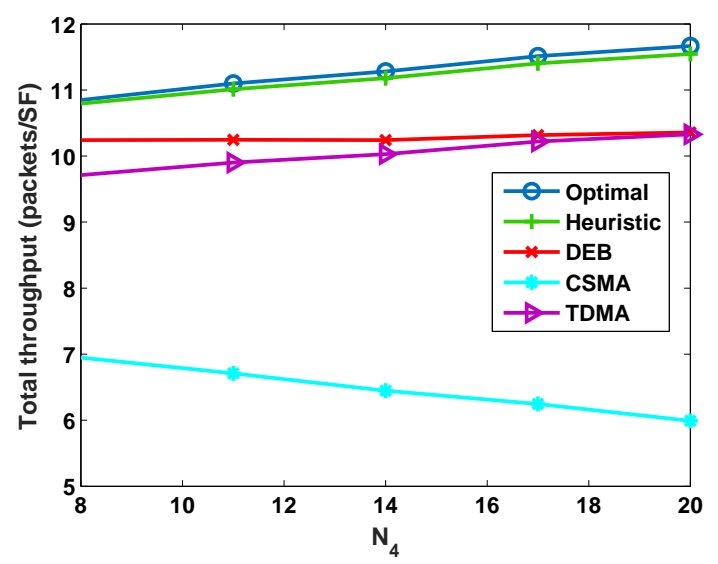

Fig. 5: Toatal throughput for $\alpha=0.1, \beta=0.9$, and $N_{1}=N_{2}=N_{3}=8$.

shows the isolation index versus the number of users belonging to SP 4 for the unbalanced scenario. As can be observed, for the proposed approaches, the isolation index is not affected by increasing $N_{4}$, whereas the isolation index of CSMA and DEB quickly drops when $N_{4}$ increases. This confirms the advantages of the proposed MAC protocols to manage isolation comparing to CSMA and DEB, that are incapable of handling a SP load imbalance situation in the network.

3) Throughput: Here, throughput is defined as the number of successfully transmitted packets per SF. For the unbalanced scenario, Figure 5 illustrates the total throughput of all SPs considering different MAC protocols. As can be observed, the proposed optimal and heuristic approaches outperform TDMA, DEB and CSMA. Furthermore, except for CSMA, it is shown that the throughput is increasing with $N_{4}$. The reason is that the number of active users increases, thus the chance of $N_{k}^{a} \ll$ $W$ decreases. On the other hand, a larger number of users leads to larger BVs, which may cause more time wasted for backoff. However, since optimal, heuristic and TDMA schemes use the belief vector and $|\alpha-\beta|=0.8$ is large, active users are more likely assigned lower BVs. But, the CSMA throughput decreases due to the higher number of collisions.

\section{CONCLUSION}

We have proposed a MDP-based MAC protocol with deterministic backoffs for a virtualized WLAN to improve both network utilization and SP isolation. This approach works as a virtual polling-based MAC, but without PF overheads. In this approach, at each SF, AP assigns unique BVs to users based on their traffic statistics and SP reservations. Such deterministic BV assignment avoids collisions among users. Numerical results confirm its efficiency in comparison with TDMA, DEB and CSMA in unsaturated networks. The performance is measured in terms of PDR, isolation index, and throughput. It is shown that this MDP-based MAC can keep isolation among different SPs regardless of their numbers of users or arrival traffic statistics. 


\section{REFERENCES}

[1] H. Wen, P. K. Tiwary, and T. Le-Ngoc, Wireless Virtualization. Springer, 2013.

[2] F. Zhang, W. He, Y. Chen, Z. Li, X. Wang, S. Chen, and X. Liu, "Thwarting Wi-Fi side-channel analysis through traffic demultiplexing," IEEE Trans. Wireless Commun., vol. 13, no. 1, pp. 86-98, Jan. 2014.

[3] L. Xia et al, "Virtual WiFi: Bring virtualization from wired to wireless," in Proc. ACM Conf. Virtual Execution Env., 2011.

[4] Y. Shoji and T. Hiraguri, "Virtualization-capable multichannel WiFi system with a coordinated downlink transmission technique," in Proc. Intl Symp. Commun. Systems, Networks Digital Signal Processing (CSNDSP), Jul. 2014, pp. 502-507.

[5] R. Kokku et al., "NVS: a virtualization substrate for WiMAX networks," in Proc. ACM conf. Mobile comput. netw., 2010.

[6] B. Shrestha, E. Hossain, and K. W. Choi, "Distributed and centralized hybrid CSMA/CA-TDMA schemes for single-hop wireless networks," IEEE Trans. Wireless Commun., vol. 13, no. 7, pp. 4050-4065, Jul. 2014.

[7] A. Checco and D. J. Leith, "Fair virtualization of 802.11 networks," IEEE/ACM Trans. Netw., Dec. 2013.

[8] "IEEE standard part 11: Wireless lan medium access control (MAC) and physical layer (PHY) specifications," Jun. 2007.

[9] Y. He and X. Ma, "Deterministic backoff: Toward efficient polling for IEEE 802.11 e HCCA in wireless home networks," IEEE Trans. Mobile Comput., vol. 10, no. 12, pp. 1726-1740, Jul. 2011.

[10] T. Holliday and A. Goldsmith, "Optimal power control and sourcechannel coding for delay constrained traffic over wireless channels," in Proc. IEEE Intl. Conf. Commun. (ICC), vol. 2. IEEE, 2002, pp. $831-835$.

[11] M. M. Nasralla and M. G. Martini, "A downlink scheduling approach for balancing QoS in LTE wireless networks," in Proc. IEEE Intl. Symp. on Personal, Indoor and Mobile Radio Commun. (PIMRC). IEEE, 2013, pp. 1571-1575.

[12] C.-N. Chuah and R. H. Katz, "Characterizing packet audio streams from internet multimedia applications," in Proc. IEEE Intl. Conf. Commun. (ICC), vol. 2. IEEE, 2002, pp. 1199-1203.

[13] C. Buratti, "Performance analysis of ieee 802.15. 4 beacon-enabled mode," IEEE Trans. Veh. Technol., vol. 59, no. 4, pp. 2031-2045, 2010.

[14] M. Derakhshani and T. Le-Ngoc, "Cognitive MAC designs: Background," in Cognitive MAC Designs for OSA Networks. Springer International Publishing, 2014, pp. 15-31.

[15] M. L. Puterman, Markov decision processes: discrete stochastic dynamic programming. John Wiley \& Sons, 2009.

[16] R. Jain, D.-M. Chiu, and W. R. Hawe, A quantitative measure of fairness and discrimination for resource allocation in shared computer system. Eastern Research Laboratory, Digital Equipment Corporation Hudson, MA, 1984. 